Leon R. Schild*, Dominik Lemke, Felix Boehm, Jens Greve, Lutz Dürselen, Marc O. Scheithauer, Thomas K. Hoffmann and Patrick J. Schuler

\title{
Force effects on anatomical structures in transoral surgery - videolaryngoscopic prototype vs. conventional direct microlaryngoscopy
}

https://doi.org/10.1515/cdbme-2020-0021

\begin{abstract}
Direct microlaryngoscopy is a frequently performed procedure in otorhinolaryngology, whereby considerable force is exerted on the patients' teeth, as well as oropharyngeal and laryngeal structures. Especially in cases of a challenging exposure of the anterior commissure, the cervical spine needs to be brought into a hyperextended position, which is not possible in every patient. Therefore, the conflict between the straight rigid microlaryngoscope and the curvature of the oropharyngeal corridor frequently results in tissue trauma with the consequence of teeth fracture, hematoma or nerve injury. We have developed the sMAC system, a hyper-angulated video laryngoscope with flexible instruments for transoral laryngeal surgery, which so far shows high feasibility in preclinical studies. Due to its curved shape it may exert less force on teeth and supraglottis as conventional direct microlaryngoscopy. We quantified the effects of these two surgical systems using flexible pressure sensors in two different scenarios: a mobile, hyperextended and an immobile cervical spine of the dummy, to simulate a challenging airway. We could demonstrate a $21 \%$ ( $40 \%$ with immobilized cervical spine) reduction of the average peak force acting on the maxillary incisors and a 55\% (65\% with immobilized cervical spine) reduction of the average intraoperative force on the supraglottis. The developed prototype applied therefore significantly less force on upper front teeth and supraglottis as compared to conventional direct microlaryngoscopy especially in the case of an immobilized cervical spine.
\end{abstract}

Keywords: force; larynx; surgery; TLM; video laryngoscope.

*Corresponding author: Leon R. Schild, Department of Otorhinolaryngology, Head and Neck Surgery, Ulm, Germany, E-mail: leon.schild@uni-ulm.de

Dominik Lemke and Lutz Dürselen, Institute of Orthopaedic Research and Biomechanics, Ulm, Germany

Felix Boehm, Jens Greve, Marc 0. Scheithauer, Thomas K. Hoffmann and Patrick J. Schuler, Department of Otorhinolaryngology, Head and Neck Surgery, Ulm, Germany; Surgical Oncology Ulm, i2SOUL Consortium, Ulm, Germany
Introduction

In transoral laser microsurgery (TLM) of the larynx - the gold standard for surgical treatment of early staged laryngeal carcinoma - the vocal cord level is adjusted by direct microlaryngoscopy. In order to obtain suitable exposure of laryngeal structures of interest, particularly the anterior commissure, a considerable force must be applied to the maxillary incisors and laryngopharynx. Furthermore, the cervical spine is brought into a hyperextended position, which is not possible in every patient due to an altered cervical spine, trismus, high BMI or a history of radiation [1]. Additionally, there are several reports about extra-laryngeal complications encountered due to the microlaryngoscopic force application, including postoperative tongue pain, transient laryngeal edema, hypoglossal palsy, dysphagia, and taste alterations in up to about $20 \%$ of the cases [2]. Dental injuries may occur in up to $2.4 \%$ of the interventions [3]. There are reports in which the upper incisors were even deliberately extracted in order to obtain a sufficient view of the anterior commissure of the larynx using a microlaryngoscope [4] . There is evidence, that a curved form of the microlaryngoscope may reduce the force applied on various anatomical structures in the head and neck area [5]. We have developed the prototype of a hyper-angulated video laryngoscope equipped with flexible surgical instruments, which shows good applicability for transoral laryngeal surgery in preclinical studies. We hypothesize that the developed surgical prototype may exert less force on front teeth and laryngopharynx in a surgical setting than conventional microlaryngoscopy. In the following, we report on a user study of the system in a simulation dummy with various angles of possible neck flexion-extension. Applied forces are quantified and compared by means of flexible pressure sensor foils.

\section{Material and methods}

The novel s-MAC system for laryngeal surgery consists of a hyperangulated C-MAC D-Blade videolaryngoscope with built-in camera 
and light source (Karl Storz, Tuttlingen, Germany) and attached dualarm flexible instrumentation. On both sides, working channels of PTFE material are fixed via rapid prototyped clips, which are inserted in the laryngoscope's catheter guide. The whole system is attached to the surgical table using a 3D-printed bracket (Figure 1). As surgical instruments we used the manually operated endoscopic tool DiLumen grasper (Lumendi, Westport, USA), which were originally designed for endoscopic tissue manipulation in the digestive tract. The instruments were shortened by the manufacturer to a length of $55 \mathrm{~cm}$ to best match their use in the area of the head and neck. These tools have a diameter of $6 \mathrm{~mm}$ can be tilted up to 90 degrees in polar angle and 360 degrees in azimuthal angle, resulting in a spherical workspace.

For microlaryngoscopy, we used the widely used Kleinsasser operating laryngoscope OP 292 (Aesculap, Tuttlingen, Germany) with its chest support. Visualization and position control were provided by the video laryngoscopic 7 -inch standard monitor, respectively a smartphone camera with an attached endoscope to smartphone adapter (SMART SCOPE, Karl Storz, Tuttlingen, Germany) and rigid endoscope $\left(0^{\circ}\right)$ for the operating laryngoscope. To measure the peak and intraoperative force exerted by the two devices on the supraglottis and the maxillary incisors, calibrated thin, flexible pressure sensors were used. We used a system and sensor type which is normally applied for intra-articular contact pressure measurement in the knee joint: the Tekscan I-Scan system with a K-Scan Type 4000 sensor $\left(2 \times 920.7 \mathrm{~mm}^{2}, 1500\right.$ psi; Tekscan, Inc., Boston, MA, USA). The sensor enables a continuous recording of the contact pressure distribution with a sampling rate of $20 \mathrm{~Hz}$. Sensor calibration and equilibration was performed in a material testing machine (Z010, Zwick, Ulm, Germany) using a customized calibration algorithm and setup, as described before [6]. The calibration supporting points were defined as seven values $(10,25,50,75,100,125,150 \mathrm{~N})$. During the calibration of the pressure measuring foils, each of these force values was hold for $30 \mathrm{sec}$, forming several plateaus. At the same time the raw values were recorded using the I-scan software. In order to assign the appropriate force value to each raw data value, the average value of the plateau was formed, and a measurement curve was interpolated using quadratic regression. The calibration of the sensors took place under the same external influences as the later preclinical study.

Prototype and operating laryngoscope were each aligned and fixed successively at vocal cord level. The projection points of raised epiglottis and maxillary incisors on the respective blade were marked. One of the two arms of each sensor was attached to these locations with double-sided adhesive tape (Figure 2).
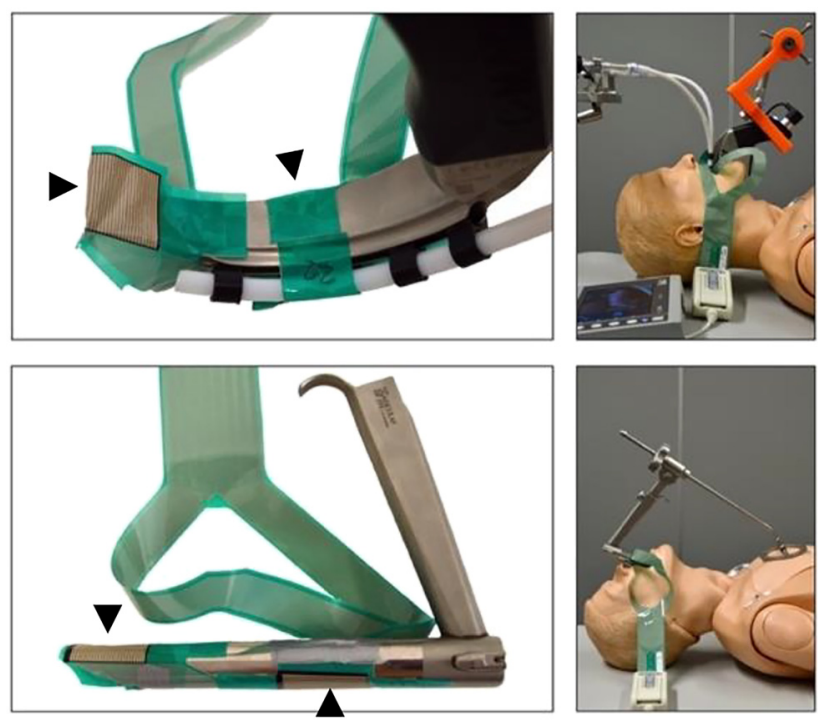

Figure 2: Experimental setup: pressure sensor foil is attached to the microlaryngoscope and surgical prototype. Sensors are marked by arrows.

The sensor was plugged into the I-Scan system and data were processed, visualized and stored via the software I-Scan 6.03 (Tekscan, Inc., Boston, MA, USA). The experiments were performed by three participants from the ENT-Clinic Ulm (senior physician, resident, medical student) with different experience in intubation and microlaryngoscopy, as the correct technique and application of force when adjusting the surgical laryngoscope is very dependent on experience. All participants applied the two devices to a simulation dummy with a mobile (scenario A) and immobilized (scenario B) cervical spine. In scenario $\mathrm{A}$, the devices were adjusted to vocal cord level in a dummy with a mobile and hyperextended cervical spine. The respective device was attached to the operating table and photo documentation was carried out to ensure correct positioning. The procedure was repeated 25 times. In scenario B, the neutral position of the cervical spine of the dummy was immobilized via cervical support (stiff neck) and the vocal cord level was adjusted 5 times.

Raw data were extracted from a force-time curve using the I-Scan software, which can be divided into two typical parts: the introductory

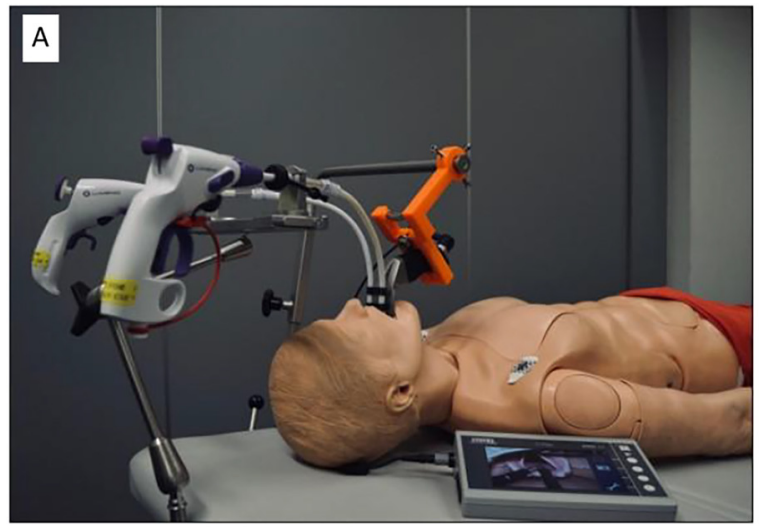

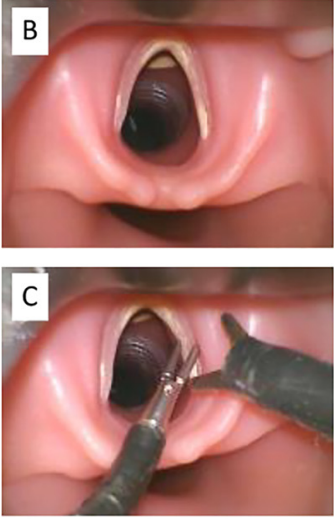

Figure 1: (A) The s-MAC surgical system. The system is attached to the operating rod through a 3D-printed bracket and flexible instruments are inserted. Representation of the (B) vocal cord level and the (C) inserted s-MAC system during a fixed cervical spine. 


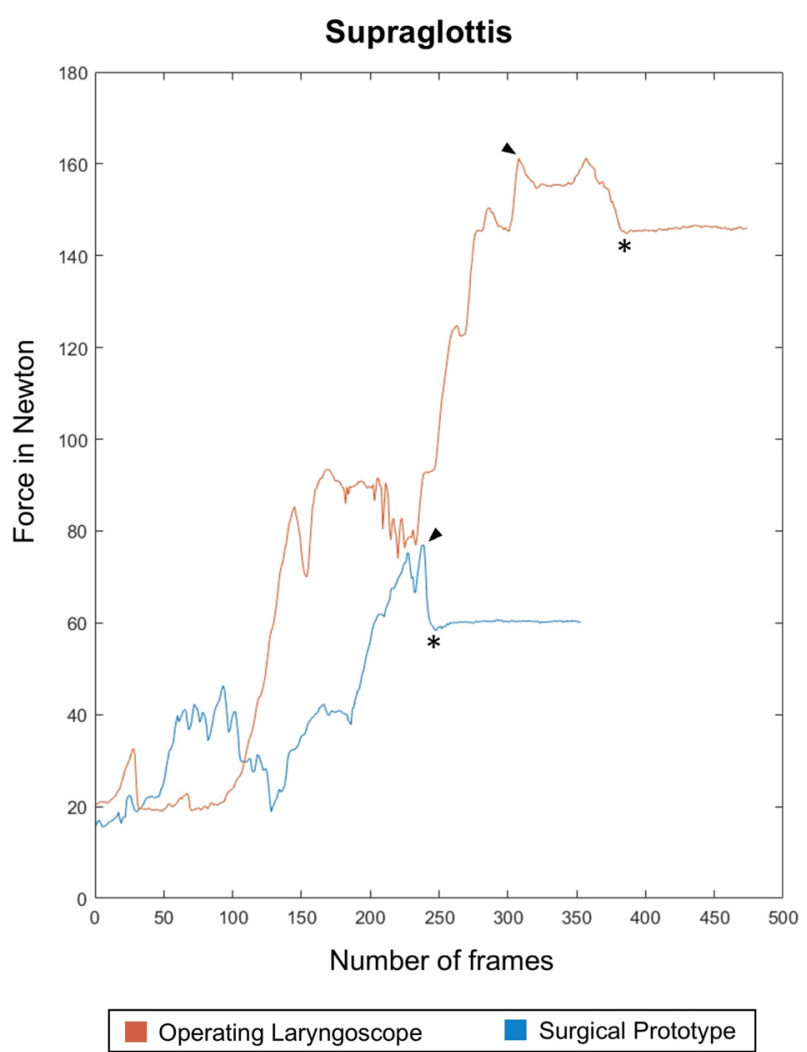

Figure 3: Exemplary force-time curve of the force effect on the supraglottis between the devices. The graph can be divided in a volatile introductory phase and an intraoperative plateau phase. Peak Force (arrows) and the begin of the intraoperative force (asterisk) are marked.

phase of the device, where volatile force swings occur, and a plateau phase, which occurs when the user locks the device in the correct position. This plateau phase reflects the long-lasting intraoperative force that is usually applied to larynx and teeth. Exemplary force-time curves for direct microlaryngoscope and prototype are shown in Figure 3.

Statistical analysis and inter-group comparisons were evaluated with the nonparametric Mann-Whitney-U-Test for independent samples and Dunn-Bonferroni post-hoc test. If not stated otherwise, results are reported as mean \pm standard error of the mean, while statistical significance was assumed for a p-value below 0.05 .

\section{Results}

The comparison of force application between the devices on the mobile dummy showed significantly lower values in peak force at the incisors $(\mathrm{p}=0.002)$ using the surgical prototype. At the larynx a significantly lower peak force ( $<<0.001)$ and significantly lower permanent intraoperative force $(p<0.001)$ was shown, while there was no statistical difference between the two groups when comparing the permanent intraoperative force on the teeth $(44.2 \mathrm{~N}$ vs. $44.3 \mathrm{~N} ; \mathrm{p}=1$ ). These data suggest that the average laryngeal intraoperative force applied was reduced by $55 \%$ using the surgical prototype, while the maximum force acting on the larynx was two times higher when using the conventional surgical laryngoscope. A moderate decrease $(10 \%)$ in the maximum force on the teeth was observed when the vocal cord level was visualized using the surgical prototype.

The measured values during immobilization of the cervical spine with cervical support (stiff neck) showed overall significant differences: peak forces on the larynx $(p<0.001)$ and teeth $(p=0.001)$, as well as average intraoperative force application on the larynx $(p<0.001)$ and teeth $(p=0.009)$ were significantly lower when using the prototype compared with the microlaryngoscope. When using the prototype instead of the microlaryngoscope, that the maximum force acting on the teeth was reduced by $39 \%$, whereas in the same comparison the prototype exerted only $35 \%$ of the permanent intraoperative force on the larynx. Comparing the force application of the prototype in mobile and immobilized cervical spine, the maximum force on teeth even decreased ( $51.9 \mathrm{~N}$ vs. $45.8 \mathrm{~N})$. The intraoperative force on the larynx remained largely unchanged ( $52.3 \mathrm{~N}$ vs. $52.2 \mathrm{~N}$ without immobilization). Only the maximum force on larynx increased by about $20 \%$ from $57.7 \mathrm{~N}$ to $70.4 \mathrm{~N}$. When using the operating laryngoscope on the immobilized dummy, added force was applied at each of the measurement points compared with mobile cervical spine: The smallest increase affected the parameter maximum force on the teeth (factor 1.14), while the maximum force on the teeth and intraoperative force on the

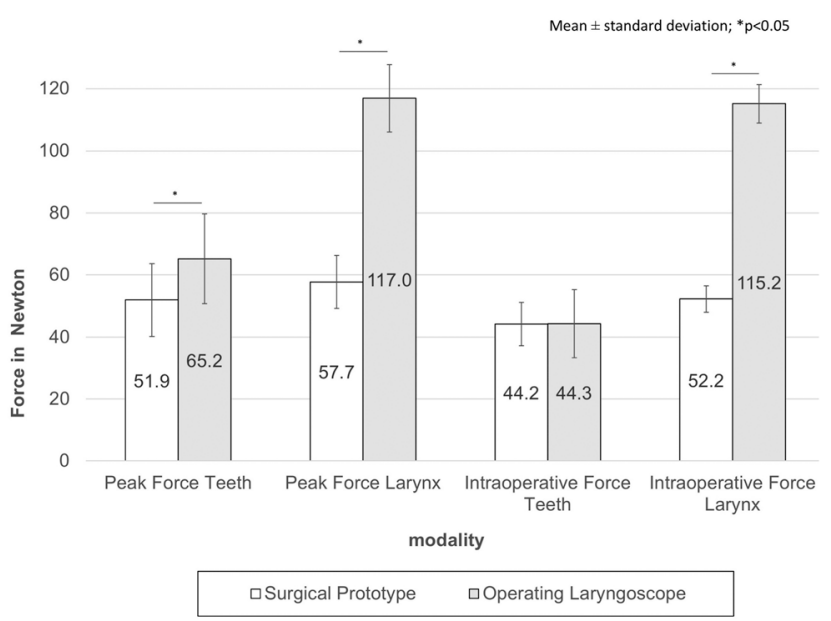

Figure 4: Comparison of contact force exerted on teeth and larynx with mobile head and neck $(n=25$, mean \pm SD). 


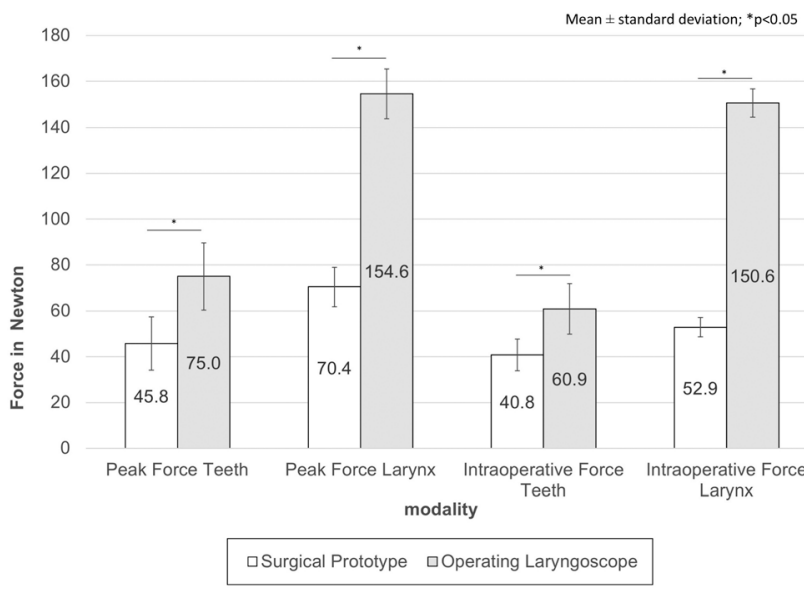

Figure 5: Comparison of contact force exerted on teeth and larynx with immobilized head and neck $(n=5$, mean \pm SD).

larynx and teeth were increased by at least 30\%. A comparison of prototype and operating laryngoscope in both scenarios is shown in Figures 4 and 5.

\section{Discussion}

We found that the force effect on upper front teeth and supraglottis in transoral surgery could be significantly reduced by using a modified video laryngoscope instead of a direct microlaryngoscope in a preclinical setting.

It is known that direct microlaryngoscopy exerts considerable force on teeth and laryngeal structures. If these are compared with the forces that occur during intubation using a video laryngoscope, a considerable reduction is shown, especially in the case of hyperangulated video laryngoscopes. However, these two scenarios have been too different to allow a reasonable comparison: the direct microlaryngoscope is kept under tension throughout the entire surgical procedure by a hyperextension of the patient's cervical spine, whereby the laryngopharynx serves as an abutment for the microlaryngoscopic blade. The video laryngoscope on the other hand, is inserted manually by an anesthetist for a few seconds to show the vocal cord level for the insertion of the endotracheal tube. To the authors' knowledge, until now no video laryngoscope has been modified for transoral laryngeal surgery. Thus, we were able to show for the first time that the advantages regarding the force application of a hyper-angulated video laryngoscope also apply in a surgical setting. The curved prototype has so far shown high feasibility of laryngeal manipulation in a simulation dummy and ex-vivo experiment. The fact that the system exerts considerably less force on teeth and supraglottis, especially in patients with difficult access to the larynx, is another valid advantage and could in the future - with narrow indications - represent an alternative to conventional transoral (laser) microsurgery. Limitations of the study are the relatively small number of repetitions as well as the testing on a simulation dummy, where the clinical use cannot be concluded 1:1.

\section{Conclusion}

Using the surgical prototype for laryngeal surgery applies significantly less force on upper front teeth and laryngopharynx compared to conventional direct microlaryngoscopy in a pre-clinical setting. Especially in patients with immobilized cervical spine this may reduce the complication rate and improve the patient's outcome and quality of life. Nevertheless, the application of the prototype must be evaluated in human body donors and clinical studies to confirm previous results.

Research funding: The author state no funding involved. Author contributions: All authors have accepted responsibility for the entire content of this manuscript and approved its submission.

Competing of interests: Authors state no conflict of interest.

Informed consent: Informed consent was obtained from all individuals included in this study.

\section{References}

1. Piazza C, Mangili S, Bon FD, Paderno A, Grazioli P, Barbieri D, et al. Preoperative clinical predictors of difficult laryngeal exposure for microlaryngoscopy: the Laryngoscore. Laryngoscope 2014;124:2561-7.

2. Feng AL, Song PC. Laryngeal force sensor: quantifying extralaryngeal complications after suspension microlaryngoscopy. Otolaryngol Head Neck Surg 2018;159:328-34.

3. Müller A, Verges L, Schleier P, Wohlfarth M, Gottschall R. The incidence of microlaryngoscopy associated complications. HNO 2002;50:1057-61.

4. Saravanappa N, Ward VM, Harries ML. Deliberate removal of incisor teeth to allow access for laryngoscopy. J Laryngol Otol 2001;115: 302-3.

5. Friedrich G, Kiesler K, Gugatschka M. Curved rigid laryngoscope: missing link between direct suspension laryngoscopy and indirect techniques?. Eur Arch Oto Rhino Laryngol 2009;266:1583-8.

6. Seitz AM, Lubomierski A, Friemert B, Ignatius A, Dürselen L. Effect of partial meniscectomy at the medial posterior horn on tibiofemoral contact mechanics and meniscal hoop strains in human knees. J of Orthopaedic Research 2012;30:93. 